\title{
Oligometastatic non-small cell lung cancer: a narrative review of stereotactic ablative radiotherapy
}

\author{
Michael Dohopolski, Puneeth Iyengar \\ Department of Radiation Oncology, UT Southwestern Medical Center, Dallas, TX, USA \\ Contributions: (I) Conception and design: All authors; (II) Administrative support: None; (III) Provision of study materials or patients: All authors; \\ (IV) Collection and assembly of data: All authors; (V) Data analysis and interpretation: All authors; (VI) Manuscript writing: All authors; (VII) Final \\ approval of manuscript: All authors.
}

Correspondence to: Puneeth Iyengar, MD, PhD. UT Southwestern Medical Center, 2280 Inwood Road, Dallas, TX, USA.

Email: puneeth.iyengar@utsouthwestern.edu.

\begin{abstract}
Non-small cell lung cancer (NSCLC) is the most common and deadly malignancy in the United States. A significant portion of these individuals can present with or later develop metastatic NSCLC (mNSCLC). These patients typically do not survive more than two to three years after diagnosis despite the use of systemic therapies; however, there are individuals with low burden mNSCLC (oligometastatic disease) who can potentially be cured with the use of aggressive local therapies—such as stereotactic ablative radiotherapy (SAbR) - in conjunction with or without systemic therapy. Oligometastatic disease represents an intermediate state prior to the development of widespread metastases. SAbR has been shown to be an effective modality for treating patients with oligometastatic NSCLC. The combination of immunotherapy and SAbR likely represents one of the most effective while still tolerable therapies in this patient population. There are other subtypes of oligometastatic disease, including oligoprogressive disease which are amenable to SAbR. The current literature supports the use of SAbR in this population to increase the time of a patient's current systemic therapy; however, there are prospective studies evaluating the efficacy of treatment on progression free survival (PFS).
\end{abstract}

Keywords: Non-small cell lung cancer (NSCLC); oligometastatic non-small cell lung cancer (mNSCLC); stereotactic body radiation therapy (SBRT); stereotactic ablative radiotherapy (SAbR); immunotherapy

Submitted Jul 14, 2020. Accepted for publication Jan 06, 2021.

doi: 10.21037/apm-20-1409

View this article at: http://dx.doi.org/10.21037/apm-20-1409

\section{Introduction}

Non-small cell lung cancer (NSCLC) is the leading cause of cancer associated mortality in the United States despite targeted screening of at-risk populations $(1,2)$. Most patients will present with advanced or metastatic disease; moreover, a large proportion of those with advanced disease will later develop distant metastases despite aggressive definitive therapy $(3,4)$. Metastatic NSCLC (mNSCLC) offers a dismal prognosis-only $5 \%$ of patients are estimated to be alive at five years. Historically, the standard of care is systemic therapy with radiotherapy often reserved for palliative intent (5). Immunotherapies, such as pembrolizamub, have been utilized more recently and have been shown to improve survival in patients with mNSCLC (6).

However, there are those with low burden mNSCLC (oligometastatic NSCLC) who can benefit from aggressive local therapy (7). Oligometastatic disease represents an intermediate state between local disease and widespread dissemination where local therapies such as surgery or radiation could provide prolonged disease-free survival or even cure (8). Several early studies showed that surgical intervention for such individuals with either pulmonary or hepatic metastases could result in extended survival beyond 5 years $(7,9)$. Stereotactic body radiation therapy (SBRT), also known as stereotactic ablative radiotherapy (SAbR), 
is a method that utilizes advanced imaging techniques, treatment optimization software, and image guidance to deliver high-dose, focused radiation. In this article, we will discuss the literature supporting the use of SAbR in the setting of oligometastatic NSCLC and explore how the combination of systemic therapy and SAbR-e.g., immunotherapy in combination with SAbR-may more effectively treat this patient population (10-12). We will also discuss the literature supporting the use of SAbR in oligoprogressive states. For this review, we performed a targeted search within PubMed and clinicaltrials.gov regarding the general topic of oligometastatic disease with a later focus on the literature surrounding oligometastatic NSCLC. Articles included were predominately published between 1990 and 2020. We present the following article in accordance with the Narrative Review reporting checklist (available at http://dx.doi.org/10.21037/apm-20-1409).

\section{Defining oligometastases}

Oligometastases, by definition, are limited in number and involved sites with a tumor biology typically lacking more virulent propensities. There have been several studies evaluating oligometastases that have slightly different criteria: 3 or fewer metastases not including the primary disease, up to 6 sites of extracranial disease including the primary, etc. $(10,11)$. The European Organisation for Research and Treatment of Cancer (EORTC) Lung Cancer Group sought to establish a single definition to be used to design and compare clinical trials: $\leq 5$ metastases in $\leq 3$ organs (not including mediastinal lymph nodes). Extensive evaluation including an 18-fluorodeoxyglucose-PET (18FDG PET/CT) and brain imaging were necessary to classify an individual as having oligometastatic disease. A solitary metastasis required biopsy confirmation or comprehensive evaluation by a multidisciplinary team (13). Most recently, the ESTRO-ASTRO consensus definition for oligometastatic disease has been announced and includes only 1-5 treatable lesions with or without a controlled primary site of disease. No assertions were made regarding number of involved organs, number of metastases within a single organ, or presence synchronous or metachronous disease as there a paucity of data to support such claims. Many supported the use of targeted high-resolution imaging including brain MRI and PET/Ct as indicated (14). Biomarkers have not yet been incorporated into these definitions. There are ongoing research utilizing microRNA, genetic profiling, circulating tumor cells, or cell-free DNA to better identify patients with oligometastatic disease (15-17).

Imaging is heavily utilized to measure the degree of metastatic disease; yet, image interpretations require clinical context. Hellman described various subtypes of oligometastatic disease aside from the traditional denovo metastases limited in number and location including metastatic disease that was once widely metastatic but then later mostly eradicated by systemic therapy (8). Guckenberger et al. recently published a consensus article that described clinical subtypes including de-novo oligometastatic disease (synchronous vs. metachronous), oligorecurrence, oligoprogression, and oligopersistence (13). Proper classification can focus future outcome analyses that will aid in personalized treatment decision-making. Results from a recent case-biased survey noted, however, the definition of oligometastatic NSCLC used by daily practitioners is more conservative than the definitions used in clinical trials. These physicians considered patients to have oligometastatic disease if they had a single metastasis with or without lymph node involvement (N0-1). This practice excludes patients who might otherwise benefit from aggressive local therapy (18).

\section{Retrospective and early prospective literature review of oligometastatic NSCLC}

Prospective evidence from Patchell et al. supported the use of surgical resection in patients with a single brain metastasis. The cohort included predominantly NSCLC histology, and outcomes showed improved local control and overall survival (OS) (19). An extensive review of the surgical literature in regards to oligometastatic NSCLC is outside the scope of this review; however, a summary of results can be seen in Patrini et al. where they discuss the utility of excising sites of mNSCLC from brain, bone, lung, and adrenal glands (20).

SAbR, alternatively, provides an efficacious noninvasive modality to treat patients with oligometastatic NSCLC. SAbR provides several benefits over surgery including very limited recovery periods that allow patients to initiate systemic therapies as soon as possible. Early retrospective data from 23 patients with newly diagnosed oligometastatic NSCLC (defined as one or two sites of disease with 22/23 undergoing PET evaluation) showed that definitive management with radiation with or without surgery was achievable. In this analysis, one patient was treated with resection alone, 6 patients were treated with surgery and radiation, and 15 were treated with "irradiation or stereotactic radiosurgery alone". Five patients survived 
beyond 36 months, and only two patients experienced grade $3+$ pneumonitis likely related to radiation (21). One of the first phase II studies evaluating the radical treatment of oligometastatic NSCLC was reported by De Ruysscher et al. in 2012. They enrolled 44 patients with mNSCLC who had $<5$ metastases at initial diagnosis that were amenable to local therapies such as surgery or radiation. A majority (97\%) had WHO performance status of $0-1$, extracranial disease $(56 \%)$, single metastasis $(87 \%)$, and received chemotherapy (95\%). Median follow-up was 27.7 months. The median OS was 16.7 months, and median progression-free survival (PFS) was 12.1 months. Three-year OS and PFS were $17.5 \%$ and $13.6 \%$, respectively (22).

Several studies have been published that provide strong support for the use of SAbR in oligometastatic NSCLC. One of the first phase II studies that evaluated the use of SBRT alone in patient with oligometastatic NSCLC ( $\leq 5$ metabolically active metastatic lesions on PET) was published by Collen et al. and looked at 17 patients who received SBRT after chemotherapy and 9 patients who received SBRT alone. Median follow-up was 16.4 months; median PFS and OS were 11.2 and 23 months, respectively. Local control was not affected by the previous use of induction chemotherapy; but use of induction chemotherapy provided a significant benefit in OS (23). Iyengar et al. and Gomez et al. were the first to publish randomized data supporting the use of SAbR in oligometastatic NSCLC. A multicenter, phase II study by Gomez et al. evaluated patients with $\leq 3$ metastatic lesions after first-line systemic therapy (4 or more cycles of platinum doublet therapy or 3 or more months of EGFR or ALK inhibitors) who were randomized to local therapy with radiation, chemoradiation, or resection with or without maintenance treatment or to maintenance treatment alone (including observation). The study was closed early after interim analysis showed the median PFS in the local therapy arm was significantly improved (11.9 months) compared to the PFS in the maintenance therapy alone arm (3.9 months). Additional results showed that the appearance of new disease sites was delayed in patients receiving local therapy. Treatment associated adverse events were similar between the two groups (11). The long-term follow-up data, published in 2019, not only confirmed a PFS benefit associated with local therapy (median PFS 14.2 vs. 4.4 months at a median follow-up of 38.8 months), but also showed a median OS benefit (41.2 vs. 17.0 months). Iyengar et al. evaluated a similar cohort of patients who previously received 4-6 cycles of first-line platinum-based chemotherapy with nonprogressive oligometastatic NSCLC (primary disease plus up to 5 metastatic sites with no more than 3 sites in the liver or lung) which did not possess EGFR or ALK mutations. In this randomized, phase II study, twenty-nine patients were analyzed. The trial was also terminated early when the interim analysis showed that patients receiving SAbR in addition to maintenance therapy had significant improvements in mPFS (9.7 vs. 3.5 months). This study differs from prior studies in that tumors lacked targetable mutations, systemic therapy was deliver sequentially, local therapy only included radiation with similar biological doses, and all patients received maintenance therapy. Extrapolation of these data from these two trials to broader populations with oligometastatic NSCLC should be tempered since only 49 and 29 patients were analyzed, and the systemic therapies received were heterogeneous and did not include immunotherapies-an important distinction as immunotherapy is included in the current standard of care for patients with locally advanced disease. Despite these limitations, these data supporting the use of local therapy (including SBRT) for oligometastatic NSCLC is compelling $(10,12)$. The results from the SABRCOMET trial were recently published. This was a phase II, randomized, multicenter study of 99 patients with oligometastatic disease $(<6$ sites of metastases, different primary histologies) who were randomized in a 2:1 fashion to standard palliative treatment or standard of care and SBRT to all sites of metastatic disease. Eighteen patients with NSCLC were included. Median follow-up was 26 months. Median OS was $28 v s .41$ months and median PFS was 6.0 and 12.0 months for the control and treatment groups, respectively. Improvements in PFS and OS were at the expense of increased toxicity and three treatmentrelated deaths (24). We await the phase III (NCT03721341) results evaluating patients with limited metastatic disease (4-10 metastatic sites from various primary histologies) that again randomized patients to maintenance therapy plus SAbR vs. maintenance therapy alone. A summary of these trials and their outcomes can be seen in Table 1 .

We also await the results of the phase III trials NRGLU-002 (NCT03137771) and SARON (NCT02417662), which are powered to detect an OS benefit in patients receiving maintenance therapy plus $\mathrm{SAbR} v s$. maintenance therapy alone. SARON is a randomized, multicenter, phase III trial analyzing patients with mutation negative oligometastatic NSCLC (1-5 lesions in up to a maximum of 3 organs) who will receive standard platinum-doublet 


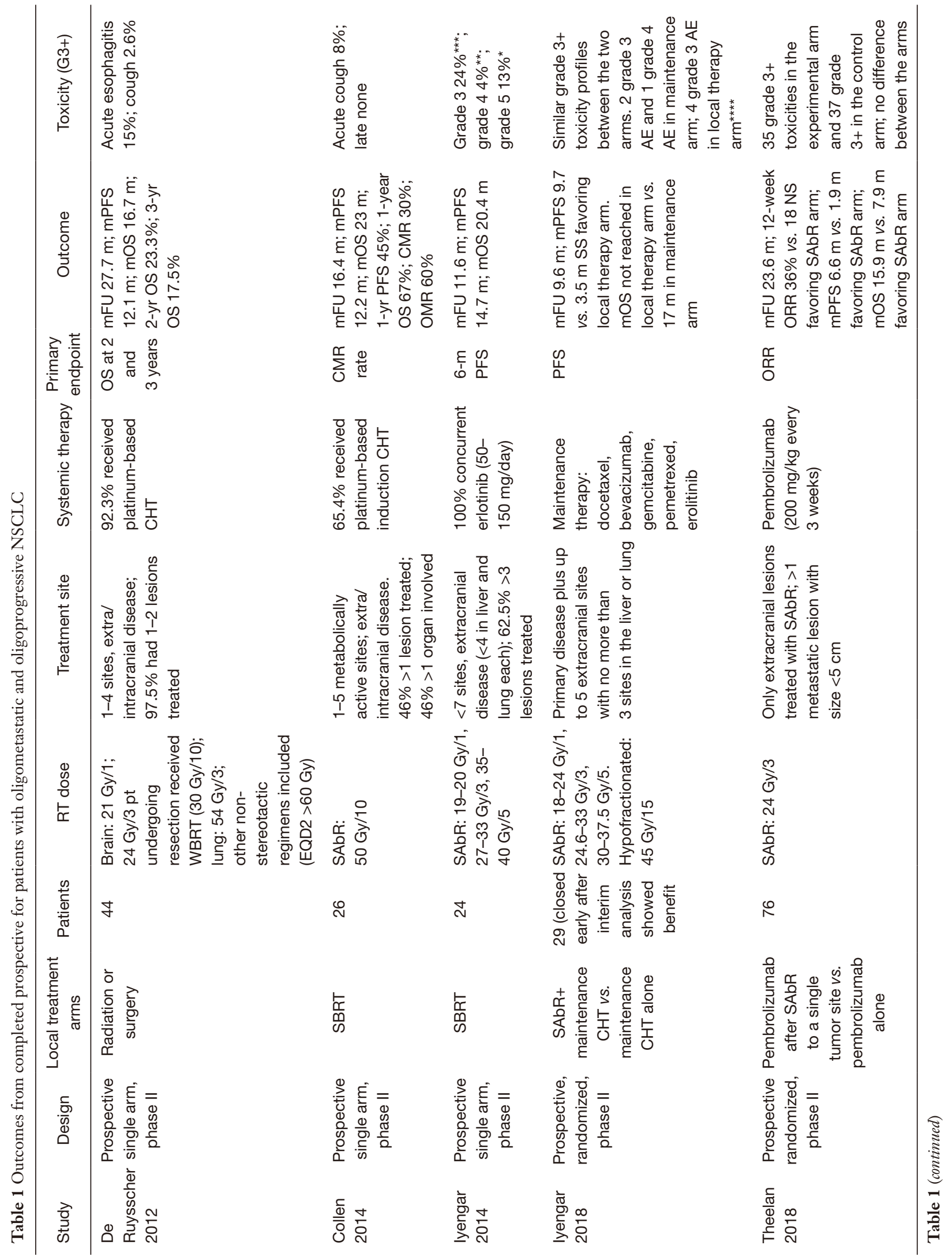




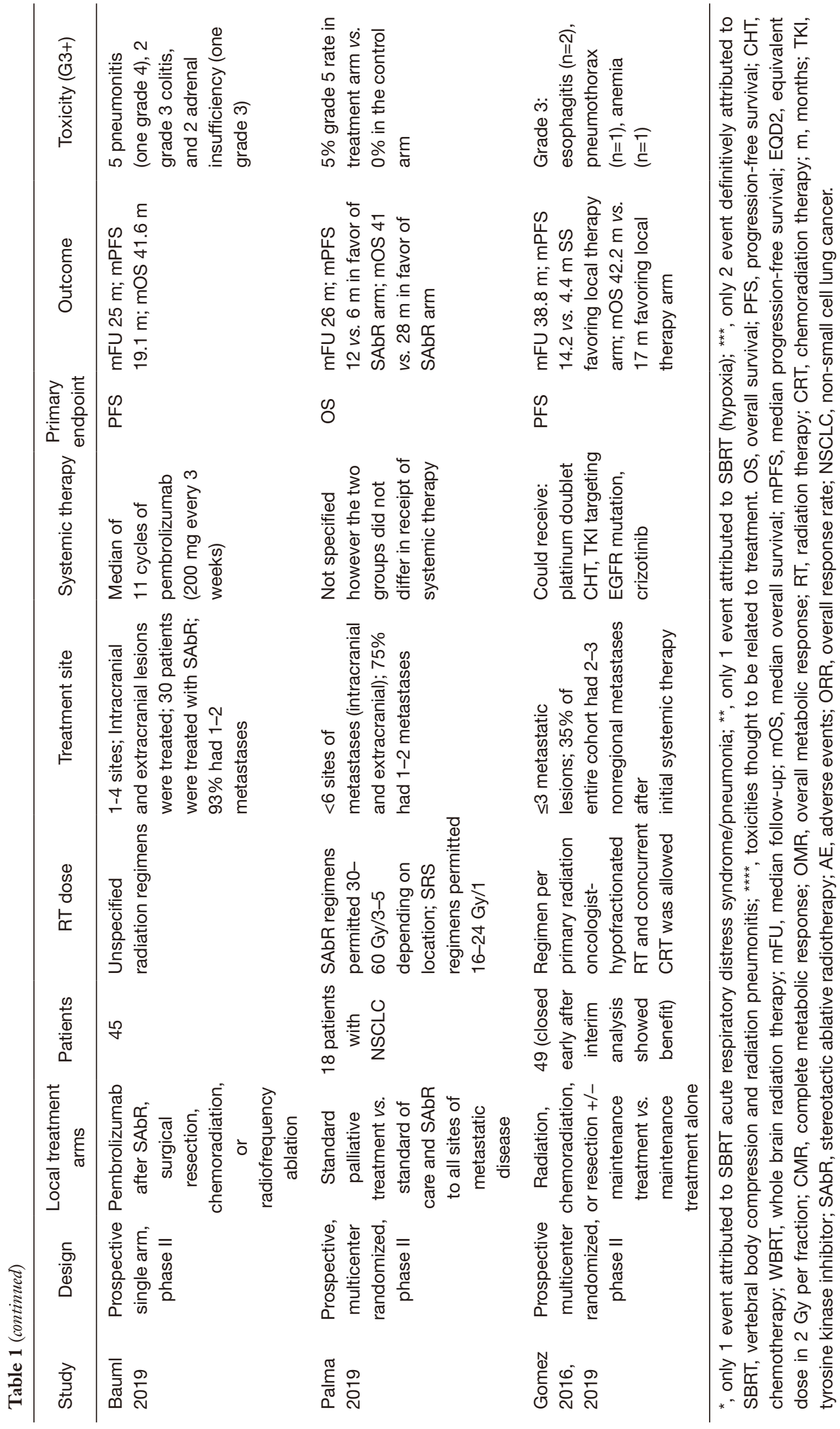


Table 2 Summary of ongoing prospective trials evaluating patients with oligoprogressive or oligometastatic NSCLC

\begin{tabular}{|c|c|c|c|c|c|c|c|c|}
\hline Study & Disease & Design & $\begin{array}{c}\text { Estimated } \\
\text { accrual }\end{array}$ & Treatment & Lesion number & Location & $\begin{array}{l}\text { Primary } \\
\text { endpoint }\end{array}$ & $\begin{array}{l}\text { Secondary } \\
\text { endpoint }\end{array}$ \\
\hline $\begin{array}{l}\text { NRG-LU-002 } \\
\text { (NCT03137771) }\end{array}$ & $\begin{array}{l}\text { Oligometastatic } \\
\text { NSCLC }\end{array}$ & $\begin{array}{l}\text { Randomized } \\
\text { multicenter } \\
\text { phase II/III }\end{array}$ & 378 & $\begin{array}{l}\text { MST vs. local } \\
\text { consolidative } \\
\text { therapy + plus } \\
\text { MST }^{\star \star}\end{array}$ & $1-3$ & Extracranial & $\begin{array}{l}\text { PFS, } \\
\text { OS }\end{array}$ & $\begin{array}{l}\text { Time to in-field } \\
\text { failure, duration } \\
\text { of maintenance } \\
\text { chemotherapy, } \\
\text { time to new lesion }\end{array}$ \\
\hline $\begin{array}{l}\text { STOP } \\
\text { (NCT02756793) }\end{array}$ & $\begin{array}{l}\text { Oligoprogressive } \\
\text { NSCLC }\end{array}$ & $\begin{array}{l}\text { Randomized } \\
\text { phase II }\end{array}$ & 54 & $\begin{array}{l}\text { SC vs. SC + } \\
\text { SAbR }\end{array}$ & $\begin{array}{l}1-5 ; 1-3 \\
\text { progressing } \\
\text { lesions; max } \\
\text { of } 3 \text { lesions in } \\
\text { single organ }\end{array}$ & $\begin{array}{l}\text { Intracranial and } \\
\text { extracranial }\end{array}$ & PFS & $\begin{array}{l}\text { OS, QoL, toxicity, } \\
\text { LC, total time on } \\
\text { chemotherapy, } \\
\text { patterns of failure }\end{array}$ \\
\hline $\begin{array}{l}\text { SABR } \\
\text { COMET } 10 \\
(\text { NCT03721341) }\end{array}$ & $\begin{array}{l}\text { Oligometastatic } \\
\text { NSCLC }\end{array}$ & $\begin{array}{l}\text { Randomized } \\
\text { multicenter } \\
\text { phase III }\end{array}$ & 159 & $\begin{array}{l}\text { SC vs. SC + } \\
\text { SAbR }\end{array}$ & $4-10$ & $\begin{array}{l}\text { Intracranial and } \\
\text { extracranial }\end{array}$ & os & $\begin{array}{l}\text { QoL, toxicity, } \\
\text { PFS, time to new } \\
\text { metastasis }\end{array}$ \\
\hline $\begin{array}{l}\text { OMEGA } \\
\text { (NCT03827577) }\end{array}$ & $\begin{array}{l}\text { Oligometastatic } \\
\text { NSCLC }\end{array}$ & $\begin{array}{l}\text { Randomized } \\
\text { phase III }\end{array}$ & 195 & $\begin{array}{l}\text { Local ablative } \\
\text { therapy vs. } \\
\text { conventional } \\
\text { treatment }\end{array}$ & $\begin{array}{l}1-3 \text {; if brain } \\
\text { involvement } \\
\text { then }<2 \text { sites } \\
<3 \mathrm{~cm}\end{array}$ & $\begin{array}{l}\text { Intracranl and } \\
\text { extracranial }\end{array}$ & OS & $N / A^{*}$ \\
\hline
\end{tabular}

*, not specified on clinicaltrials.gov; ${ }^{* *}$, MST can include immunotherapy. RT, radiation; OS, overall survival; PFS, progression-free survival; LC, local control; SACT, systemic anti-cancer therapy; SAbR, stereotactic ablative radiotherapy; MST, maintenance systemic therapy; QoL, quality of life; SC, standard of care; TKI, tyrosine kinase inhibitor.

chemotherapy or standard chemotherapy followed by SAbR to their primary tumor and additional metastatic sites (25). NRG-LU-002, a phase II/III, randomized, multicenter trial is also evaluating patients with oligometastatic NSCLC with 1-3 sites of disease after four cycles of first line/induction systemic therapy (including pembrolizumab), excluding the primary site, who have exhibited responsive or stable disease to receive either SAbR or surgery to all sites of disease or continued maintenance systemic therapy alone (26). OMEGA (NCT03827577) trial is also a recently opened randomized phase III trial that is randomizing patients with oligometastatic NSCLC to standard of care medical treatment (control) $v s$. local therapy (surgical resection in primary site amenable and SAbR or radiofrequency ablation to the metastatic sites) and standard medical therapy. A summary of these ongoing prospective trials can be seen in
Table 2.

\section{Immunotherapy and SAbR in oligometastatic NSCLC}

Immunotherapy has been successfully utilized in the treatment of mNSCLC. KEYNOTE 24, which randomized patients to pembrolizumab versus investigator's choice of platinum-based chemotherapy, showed improved OS for patients who received pembrolizumab (median OS 30.0 vs. 14.2 months, respectively) (6). Moreover, adjuvant durvalumab following definitive chemoradiation was shown to significantly improve median PFS from 5.6 to 16.8 months when compared to definitive chemoradiation alone for patients with locally advanced NSCLC $(27,28)$. There have been multiple studies that have investigated SBRT and immunotherapy. SBRT is thought to aid immunotherapy through multiple mechanisms including $\mathrm{T}$ cell exhaustion by 
tumor debulking. Radiation can also upregulate cell surface markers and engage the innate immune system (6,29-33). There are limited published prospective studies focused on the utility of immunotherapy and SBRT in patients with oligometastatic NSCLC. Bauml et al. published the results from a phase II, single arm study where 45 patients with oligometastatic NSCLC (defined as less than five metastases) previously treated with SAbR, surgical resection, chemoradiation, or radiofrequency ablation received pembrolizumab. Patients received a median of 11 cycles of pembrolizumab. Median follow-up was 25.0 months, and median PFS was 19.1 months (statistically significant improvement compared to a historical control of 6.6 months). Median OS was 41.6 months. Five episodes of pneumonitis (one grade 4), two episodes of grade 3 colitis, and two episodes of adrenal insufficiency (one grade 3) were reported (34). An additional phase II trial from the Netherlands randomized 76 patients with mNSCLC to receive pembrolizumab after SBRT to a single tumor site or pembrolizumab alone. Objective response rate (ORR) at 12 weeks was $18 \%$ vs. $36 \%$, and median PFS was 1.9 vs. 6.6 months in the control vs. experimental arm. Median OS was improved in patients that received SBRT prior to pembrolizumab (15.9 vs. 7.6 months) (35). NRG-LU-002 will be one of the few phase III trials that will have allowed patients to have received immunotherapy and thus will provide outcomes more representative of the current therapeutic climate.

\section{Oligoprogressive NSCLC}

As mentioned in Guckenberger et al. recently published consensus article, there are multiple subtypes of oligometastatic disease. Oligoprogression defines a state where patients may have multiple sites of stable disease with only a few foci progressing through the current therapy. SAbR offers a means to control those limited progressive sites and allows patients to continue with their current maintenance regimen. In a retrospective series, Gan et al. evaluated 33 patients who progressed while receiving crizotinib who were then considered for locally ablative therapy in less than 5 sites of progressive disease. Twentynine oligoprogressive sites were treated with radiation. PFS outcomes favored those who received locally ablative therapy over those who were not eligible (14 vs. 7.2 months, respectively) (36).

Iyengar et al. published the results from a single arm, phase II study that included 24 patients with mNSCLC who had no more than 6 sites of extracranial disease after failed platinum-based systemic therapy. Patients received SBRT and concurrent erlotinib. Fifteen of the 24 patients enrolled were treated to two or more sites in a variety of locations. Patients were more likely to relapse distantly compared to locally (defined as location treated with SBRT). Treating new sites of progression allowed patients to continue erlotinib for an additional 6-9 months. Median PFS and OS were 14.7 and 20.4 months, respectively; median follow-up was 11.6 months (37). These results were superior to prior historical controls which reported 2 4 months PFS and 6-9 months OS (38). We await the results of the randomized STOP (NCT02756793) and HALT (NCT03256981) trials which are evaluating the efficacy of locally ablative therapy for patients with oligoprogressive NSCLC. STOP is a multicenter phase II trial where patients with oligoprogressive NSCLC (who have a maximum of three lesions in a single organ including the brain and a total maximum of five lesions) are randomized to standard of care systemic therapy plus SABR to all sites of progressive disease plus the continuation of their current systemic therapy or standard of care systemic therapy. HALT is a multicenter phase II/ III trial aimed to recruit 110 patients with mutation positive advanced NSCLC with oligoprogressive disease receiving TKI therapy. The experimental arm will include patients receiving SABR to a maximum of three extracranial sites while they continue their initial TKI therapy.

\section{Conclusions}

Oligometastases represent a state of limited systemic disease. It is important that we correctly identify such individuals since aggressive intervention may result in prolonged survival. Unfortunately, more conservative definitions of oligometastatic disease are being utilized in daily practice and thus are excluding eligible patients from optimal treatment. An official definition has yet to be established; most studies cited above limited the number of disease foci to less than 5-6 with only a limited number of disease sites (1-3 sites). The definition of oligometastatic disease has evolved since Hellman's original editorial as distinctions between de-novo oligometastatic disease (synchronous vs. metachronous), oligoprogression, etc. have been made. Appropriately classifying patients will aid in targeted clinical research, which in the future, may include multimodality approaches such as combining immunotherapy with SAbR. 


\section{Acknowledgments}

Funding: None.

\section{Footnote}

Provenance and Peer Review: This article was commissioned by the Guest Editors (Simon Lo, Michael Milano, Tithi Biswas, Charles Simone) for the series "OligometastasisFallacy or Real Deal?" published in Annals of Palliative Medicine. The article has undergone external peer review.

Reporting Checklist: The authors have completed the Narrative Review reporting checklist. Available at http:// dx.doi.org/10.21037/apm-20-1409

Conflicts of Interest: Both authors have completed the ICMJE uniform disclosure form (available at http://dx.doi. org/10.21037/apm-20-1409). The series "OligometastasisFallacy or Real Deal?" was commissioned by the editorial office without any funding or sponsorship. The authors have no conflicts of interest to declare.

Ethical Statement: The authors are accountable for all aspects of the work in ensuring that questions related to the accuracy or integrity of any part of the work are appropriately investigated and resolved.

Open Access Statement: This is an Open Access article distributed in accordance with the Creative Commons Attribution-NonCommercial-NoDerivs 4.0 International License (CC BY-NC-ND 4.0), which permits the noncommercial replication and distribution of the article with the strict proviso that no changes or edits are made and the original work is properly cited (including links to both the formal publication through the relevant DOI and the license). See: https://creativecommons.org/licenses/by-ncnd $/ 4.0 /$.

\section{References}

1. Aberle DR, Adams AM, Berg CD, et al. Reduced lungcancer mortality with low-dose computed tomographic screening. N Engl J Med 2011;365:395-409.

2. Institute NNC. Cancer Facts \& Figures 2020. CA Cancer J Clin 2020;1-76.

3. Bradley JD, Paulus R, Komaki R, et al. Standard-dose versus high-dose conformal radiotherapy with concurrent and consolidation carboplatin plus paclitaxel with or without cetuximab for patients with stage IIIA or IIIB non-small-cell lung cancer (RTOG 0617): A randomised, two-by-two factorial phase 3 study. Lancet Oncol 2015;16:187-99.

4. Antonia SJ, Villegas A, Daniel D, et al. Durvalumab after Chemoradiotherapy in Stage III Non-Small-Cell Lung Cancer. N Engl J Med 2017;377:1919-29.

5. NCCN. Non-Small Cell Lung Cancer [Internet] 2020 [cited 2020 Jul 4]. Available online: https://www.nccn.org/ professionals/physician_gls/pdf/nscl_blocks.pdf

6. Reck M, Rodríguez-Abreu D, Robinson AG, et al. Updated analysis of KEYNOTE-024: Pembrolizumab versus platinum-based chemotherapy for advanced nonsmall-cell lung cancer with PD-L1 tumor proportion score of 50\% or greater. J Clin Oncol 2019;37:537-46.

7. Pastorino U, Buyse M, Friedel G, et al. Long-term results of lung metastasectomy: Prognostic analyses based on 5206 cases. J Thorac Cardiovasc Surg 1997;113:37-49.

8. Hellman S, Weichselbaum RR. Oligometastases. J Clin Oncol 1995;13:8-10.

9. Hughes KS, Rosenstein RB, Songhorabodi S, et al. Resection of the liver for colorectal carcinoma metastases - A multi-institutional study of long-term survivors. Dis Colon Rectum 1988;31:1-4.

10. Iyengar P, Wardak Z, Gerber DE, et al. Consolidative radiotherapy for limited metastatic non-small-cell lung cancer: A phase 2 randomized clinical trial. In: JAMA Oncol 2018;4:e173501.

11. Gomez DR, Blumenschein GR, Lee JJ, et al. Local consolidative therapy versus maintenance therapy or observation for patients with oligometastatic non-smallcell lung cancer without progression after first-line systemic therapy: a multicentre, randomised, controlled, phase 2 study. Lancet Oncol 2016;17:1672-82.

12. Gomez DR, Tang C, Zhang J, et al. Local consolidative therapy vs. Maintenance therapy or observation for patients with oligometastatic non-small-cell lung cancer: Long-term results of a multi-institutional, phase II, randomized study. J Clin Oncol 2019;37:1558-65.

13. European Society for Radiotherapy and Oncology and European Organisation for Research and Treatment of Cancer. MA25.02 Searching for a Definition of Synchronous Oligometastatic (sOMD)-NSCLC: A Consensus from Thoracic Oncology Experts. J Thorac Oncol 2018;13:S446.

14. Lievens, Y. Guckenberger, M. Gomez, D, et al. Defining 
oligometastatic disease from a radiation oncology perspective: An ESTRO-ASTRO consensus document. Radiother Oncol 2020;148:157-66.

15. Neal JW, Gainor JF, Shaw AT. Developing biomarkerspecific end points in lung cancer clinical trials. Nat Rev Clin Oncol 2015;12:135-46.

16. Lussier YA, Xing HR, Salama JK, et al. MicroRNA Expression Characterizes Oligometastasis(es). Blagosklonny M V., editor. PLoS One 2011;6:e28650.

17. Barnum KJ, Weiss SA. Prognostic and Predictive Biomarkers in Oligometastatic Disease. Cancer J 2020;26:100-7.

18. Hendriks LEL, Dooms C, Berghmans T, et al. Defining oligometastatic non-small cell lung cancer: A simulated multidisciplinary expert opinion. Eur J Cancer 2019;123:28-35.

19. Patchell RA, Tibbs PA, Walsh JW, et al. A Randomized Trial of Surgery in the Treatment of Single Metastases to the Brain. N Engl J Med 1990;322:494-500.

20. Patrini D, Panagiotopoulos N, Bedetti B, et al. Surgical approach in oligometastatic non-small cell lung cancer. Ann Transl Med 2018;6:93.

21. Khan AJ, Mehta PS, Zusag TW, et al. Long term disease-free survival resulting from combined modality management of patients presenting with oligometastatic, non-small cell lung carcinoma (NSCLC). Radiother Oncol 2006;81:163-7.

22. De Ruysscher D, Wanders R, Van Baardwijk A, et al. Radical treatment of non-small-cell lung cancer patients with synchronous oligometastases: Long-term results of a prospective phase II trial (Nct01282450). J Thorac Oncol 2012;7:1547-55.

23. Collen C, Christian N, Schallier D, et al. Phase II study of stereotactic body radiotherapy to primary tumor and metastatic locations in oligometastatic nonsmall-cell lung cancer patients. Ann Oncol 2014;25:1954-9.

24. Palma DA, Olson R, Harrow S, et al. Stereotactic ablative radiotherapy versus standard of care palliative treatment in patients with oligometastatic cancers (SABRCOMET): a randomised, phase 2, open-label trial. Lancet 2019;393:2051-8.

25. Conibear J, Chia B, Ngai Y, et al. Study protocol for the SARON trial: A multicentre, randomised controlled phase III trial comparing the addition of stereotactic ablative radiotherapy and radical radiotherapy with standard chemotherapy alone for oligometastatic non-small cell lung cancer. BMJ Open 2018;8:e20690.

26. Maintenance Chemotherapy With or Without Local
Consolidative Therapy in Treating Patients With Stage IV Non-small Cell Lung Cancer - Full Text View - ClinicalTrials.gov [Internet]. [cited 2020 Apr 17]. Available online: https://clinicaltrials.gov/ct2/show/ NCT03137771? term=NRG-LU002\&rank=1

27. Antonia SJ, Villegas A, Daniel D, et al. Overall Survival with Durvalumab after Chemoradiotherapy in Stage III NSCLC. N Engl J Med 2018;379:2342-50.

28. Gray JE, Villegas A, Daniel D, et al. Three-Year Overall Survival with Durvalumab after Chemoradiotherapy in Stage III NSCLC-Update from PACIFIC. J Thorac Oncol 2020;15:288-93.

29. Lin AJ, Roach M, Bradley J, et al. Combining stereotactic body radiation therapy with immunotherapy: Current data and future directions. Transl Lung Cancer Res 2019;8:107-15.

30. Huang AC, Postow MA, Orlowski RJ, et al. T-cell invigoration to tumour burden ratio associated with antiPD-1 response. Nature 2017;545:60-5.

31. Wherry EJ. T cell exhaustion. Nat Immunol 2011;12:492-9.

32. Sharabi AB, Lim M, DeWeese TL, et al. Radiation and checkpoint blockade immunotherapy: Radiosensitisation and potential mechanisms of synergy. Lancet Oncol 2015;16:e498-509.

33. Chakraborty M, Abrams SI, Coleman CN, et al. External beam radiation of tumors alters phenotype of tumor cells to render them susceptible to vaccine-mediated T-cell killing. Cancer Res 2004;64:4328-37.

34. Bauml JM, Mick R, Ciunci C, et al. Pembrolizumab after Completion of Locally Ablative Therapy for Oligometastatic Non-Small Cell Lung Cancer: A Phase 2 Trial. JAMA Oncol 2019;5:1283-90.

35. Theelen WSME, Peulen HMU, Lalezari F, et al. Effect of Pembrolizumab after Stereotactic Body Radiotherapy vs. Pembrolizumab Alone on Tumor Response in Patients with Advanced Non-Small Cell Lung Cancer: Results of the PEMBRO-RT Phase 2 Randomized Clinical Trial. JAMA Oncol 2019;5:1276-82.

36. Gan GN, Weickhardt AJ, Scheier B, et al. Stereotactic radiation therapy can safely and durably control sites of extra-central nervous system oligoprogressive disease in anaplastic lymphoma kinase-positive lung cancer patients receiving crizotinib. Int J Radiat Oncol Biol Phys 2014;88:892-8.

37. Iyengar P, Kavanagh BD, Wardak Z, et al. Phase II trial of stereotactic body radiation therapy combined with erlotinib for patients with limited but progressive 
metastatic non-small-cell lung cancer. J Clin Oncol 2014;32:3824-30.

38. Tassinari D, Scarpi E, Sartori S, et al. Noninferiority

Cite this article as: Dohopolski M, Iyengar P. Oligometastatic non-small cell lung cancer: a narrative review of stereotactic ablative radiotherapy. Ann Palliat Med 2021;10(5):5944-5953. doi: 10.21037/apm-20-1409
Trials in Second-Line Treatments of Nonsmall Cell Lung Cancer. Am J Clin Oncol 2012;35:593-9. 